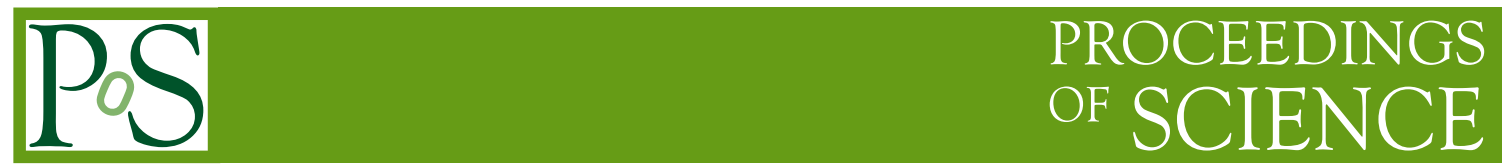

\title{
Dark Matter searches at LHC
}

\section{R. Bellan}

Università degli Studi di Torino and INFN

E-mail: riccardo.bellan@cern.ch

\section{U. De Sanctis}

Università degli Studi di Udine and INFN

E-mail: umbertoecern.ch

The origin of the Dark Matter (DM) is a puzzle that particle physics is trying to understand. Physics at the collider has a unique potentential to produce the DM candidate in laboratory. In this contribution we present the latest results of the searches for DM at the ATLAS and CMS experiments, using $\sqrt{s}=7 \mathrm{TeV}$ and $\sqrt{s}=8 \mathrm{TeV}$ data. Emphasis is given to the arguments addressed in the discussion session, in particular on the models used to interpret the experimental results.

VI Italian workshop on p-p physics at the LHC,

8-10 May 2013

Acquario di Genova, Ponte Spinola, Area Porto Antico, Genova, Italy 


\section{Introduction}

Dark matter (DM) is required to explain numerous astrophysical measurements. However, since none of the known Standard Model (SM) particles are adequate DM candidates, the existence of a new particle is hypothesised, with properties suitable to explain the astrophysical measurements. One of the best candidates for DM is a stable weakly interacting massive particle (WIMP). These particles may be pair-produced at the LHC, provided their mass is less than half the parton centre of mass energy. These particles are expected to couple to SM particles through a generic weak interaction, which could be the known weak interaction of the SM or a new type of interaction. Several new particle physics models designed to solve the hierarchy problem also predict WIMPs.

Because WIMPs do not interact with the detector material, their production leads to signatures with missing transverse momentum. The final states with one jet or a photon originating from initial/final state radiation (ISR/FSR) provide a powerful signature sensitive to DM scenarios. In model independent approaches, the interaction between the DM and SM particles is usually assumed to be mediated by a particle too heavy to be produced directly, such that the resulting interaction can be treated as a contact interaction $[1,2]$. Within such models, g the interaction between DM and SM particles are described by two parameters, the mass of the DM candidate $m_{\chi}$ and a suppression scale $\Lambda^{1}$, where the DM candidate is assumed to be a Dirac fermion. Several operators that describe this interaction can be written depending on the nature of the couplings (e.g. scalar, vectorial, axial, etc.). In this framework it is possible to compare the results coming from direct, indirect and collider experiments [3]. The latter can be then used to set limits on the parameters $m_{\chi}$ and $\Lambda$ and also on the DM-nucleon scattering cross-section as a function of the DM candidate mass $m_{\chi}$.

\section{ATLAS and CMS Results}

We presented the results from the ATLAS and CMS Collaborations. Both experiments have the analysis of mono-photon $[4,5]$ and mono-jet $[6,7]$ topologies with the additional requirement of a large missing transverse momentum. Currently, CMS reports results for the entire $\sqrt{s}=8 \mathrm{TeV}$ data-set $\left(L_{\text {int }}=19.5 \mathrm{fb}^{-1}\right)$ for the mono-jet topology, while ATLAS used half $\left(L_{\text {int }}=10.5 \mathrm{fb}^{-1}\right)$ of the available data-set. For the mono-photon final state, both experiments analysed the $\sqrt{s}=7 \mathrm{TeV}$ data-set $\left(L_{\text {int }}=5 \mathrm{fb}^{-1}\right)$ only.

The search strategies for both topologies are similar for the two experiments. They require the presence of significant missing transverse momentum balanced by a hard- $p_{T}$ jet (or photon), possibly accompanied by an additional jet close to the leading- $p_{T}$ jet (or photon). Other activities in the events are vetoed (jets and leptons). For more details see [4, 5, 6, 7].

For the mono-jet analysis, the observed and expected limits at the 90\% CL on the DM-nucleon scattering cross section are shown in Fig. 1. We displays CMS limits because they are the most updated ones, and hence more stringent. Comparing data-sets with the same size, ATLAS and CMS have similar performance. In figure 2 we show the 90\% CL upper limits on the nucleon-DM cross section as a function of $m_{\chi}$, for the mono-photon analyses. The searches at the colliders have

\footnotetext{
${ }^{1}$ In ATLAS papers $\Lambda$ is named $M_{*}$
} 
more stringent limits than direct and indirect experiments, in the low $m_{\chi}$ region $(1-4 \mathrm{GeV})$, for spin-independent interactions, and up to $300 \mathrm{GeV}$, for spin-dependent interactions.
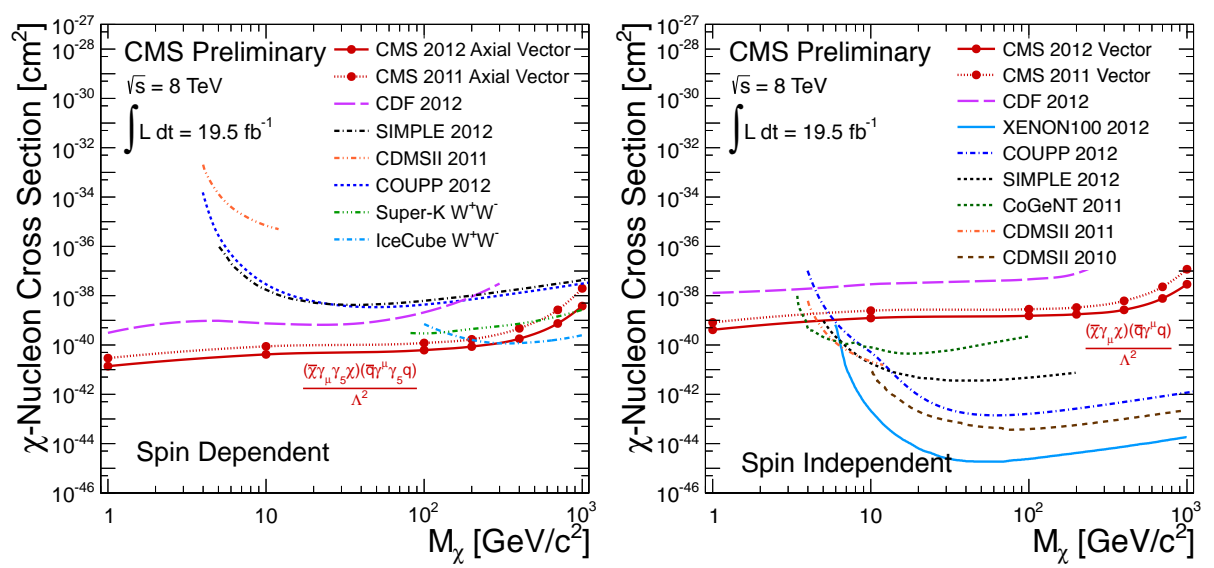

Figure 1: $90 \%$ CL upper limits on the DM-nucleon cross section versus DM candidate mass $m_{\chi}$, for the mono-jet search. The results from several experiments refer to the axial-vector (left) and vector effective operators (right).

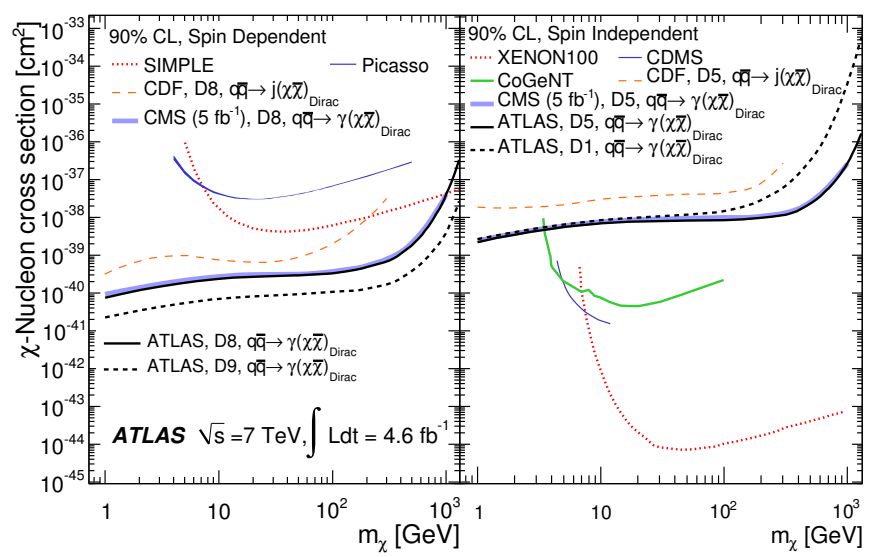

Figure 2: $90 \% \mathrm{CL}$ upper limits on the DM-nucleon cross section as a function of the DM candidate mass $m_{\chi}$, for the mono-photon analysis. The results from several experiments refer to the spin-dependent (left) and spin-independent (right) interactions.

\section{Discussion}

Several experiments $[8,9,10,11,12,13,14]$ that look for direct or indirect DM detection have discrepancies between data and theoretical predictions, that may point towards a possible interpretation of the DM as a new particle. Recently, several constraints have been set from direct and indirect DM searches and from pair production at the LHC. We believe that the LHC physics 
program should put the DM search as one of its top priorities. The LHC experiments have already produced a large number of results in final states that can be interpreted in the context of the DM search. On a fertile ground there are the searches developed to find Super-Symmetry (SUSY), or the decay of the Higgs in invisible particle. A systematic approach must be adopted to depict the characteristics of the DM particle candidate. The interpretation of the experimental results is a hot topic for this subject, as the paradigm at the basis of the effective theory $(\sqrt{\hat{s}}<<M)$ may be broken at the LHC scale, especially when LHC will move to $13-14 \mathrm{TeV}$ in the centre of mass energy. A new model or a set of models, following the Simplified-Models (SMS) approach used for SUSY $[15,16]$, is needed to serve as the starting point for building more complex theories and for their comparison with the experimental results. This effort to be effective needs close collaboration between the theory and experimental communities, which will be possible only if DM search will become a top priority at the LHC.

\section{References}

[1] J. Goodman, M. Ibe, A. Rajaraman, W. Shepherd, T. Tait, H. Yu, Constraints on Dark Matter from Colliders, Phys.Rev.D82:116010,2010, arXiv:1008.1783[hep-ph].

[2] P. Fox, R. Harnik, J. Kopp, and Y. Tsai, Missing Energy Signatures of Dark Matter at the LHC, FERMILAB-PUB-11-478-T, arXiv:1109.4398[hep-ph].

[3] Y. Bai, P. Fox, R. Harnik, The Tevatron at the frontier of Dark Matter direct detection, JHEP12 (2010) 048, arXiv:1005.3797[hep-ph].

[4] ATLAS Collaboration, Search for Dark Matter Candidates and Large Extra Dimensions in Events with a Photon and Missing Transverse Momentum in pp Collision Data at $\sqrt{s}=7 \mathrm{TeV}$ with the ATLAS Detector, PRL 110 (2013) 011802.

[5] CMS Collaboration, Search for Dark Matter and Large Extra Dimensions in pp Collisions Yielding a Photon and Missing Transverse Energy, Phys. Rev. Lett. 108, 261803 (2012).

[6] ATLAS Collaboration, Search for New Phenomena in Monojet plus Missing Transverse Momentum Final States using $10 \mathrm{fb}^{-1}$ of pp Collisions at $\sqrt{s}=8 \mathrm{TeV}$ with the ATLAS detector at the LHC, ATLAS-CONF-2012-147.

[7] CMS Collaboration, Search for new physics in monojet events in pp collisions at $\sqrt{s}=8 \mathrm{TeV}$, CMS-PAS-EXO-12-048.

[8] C. Weniger, A Tentative Gamma-Ray Line from Dark Matter Annihilation at the Fermi Large Area Telescope, JCAP 1208 (2012) 007, arXiv:1204.2797[hep-ph].

[9] M. Ackermann et al., Phys. Rev. Lett., 108, 011103 (2012).

[10] O. Adriani et al., Nature 458 (7238), 607-609 (2009).

[11] R. Bernabei et al., Eur. Phys. J. C 56, 333-355 (2008).

[12] CDMS Collaboration, Dark Matter Search Results Using the Silicon Detectors of CDMS II, arXiv:1304.4279[hep-ex]

[13] COGENT Collaboration, Results from a Search for Light-Mass Dark Matter with a p-Type Point Contact Germanium Detector, Phys. Rev. Lett. 106, 131301 (2011) 
[14] AMS Collaboration and M. Aguilar, First result from the alpha magnetic spectrometer on the international space station: Precision measurement of the positron fraction in primary cosmic rays of 0.5-350 GeV, Phys. Rev. Lett. 110, 141102 (2013).

[15] J. Alwall, P. Schuster, and N. Toro, Simplified models for a first characterization of new physics at the LHC, Phys. Rev. D79 (2009) 075020.

[16] LHC New Physics Working Group Collaboration, Simplified models for LHC new physics searches, J. Phys. G39 (2012) 105005. 